\title{
DEVELOPMENT OF AN ENGINEERING METHODOLOGY FOR THERMAL ANALYSIS OF PROTECTED STRUCTURAL MEMBERS IN FIRE
}

\author{
Hong Liang, Stephen Welch* and José L. Torero \\ BRE Centre for Fire Safety Engineering, University of Edinburgh, \\ King's Buildings, Edinburgh, EH9 3JL, United Kingdom \\ *(Corresponding author: E-mail: s.welch@ed.ac.uk)
}

Received: 25 February 2008; Revised: 6 March 2009; Accepted: 11 March 2009

\begin{abstract}
A novel CFD-based methodology for thermal analysis of protected steelwork in fire has been developed to overcome the limitations of existing methodologies. This is a generalised quasi-3D approach with computation of a "steel temperature field" parameter in each computational cell. It accommodates both uncertainties in the input parameters and possible variants to the specification by means of many simultaneous thermal calculations. A framework for the inclusion of temperature/time-dependent thermal properties, including the effects of moisture and intumescence, has been established. The method has been implemented as the GeniSTELA submodel within SOFIE RANS CFD code. The model is validated with respect to the BRE large compartment fire tests. Sensitivity studies reveal the expected strong dependencies on certain properties of thermal protection materials. The computational requirements are addressed to confirm the practicability of the tool in simultaneously running a large number of parametric variants. Ultimately, the steel temperature field prediction provided by GeniSTELA provides far more flexibility in assessing the thermal response of structures to fire than has been available hitherto; hence it could be further used for the structural response analysis, demonstrating the potential practical use of the method to improve the efficiency and safety of the relevant structural fire safety design.
\end{abstract}

Keywords: CFD; thermal analysis; quasi-3D; GeniSTELA; SOFIE

\section{INTRODUCTION}

Increasing interest in assessing the performance of structures in fire is driving the development of an array of modelling methodologies to be used in fire safety engineering design. Whilst traditionally most code-based design has been based on simple calculations, referencing measured fire performance in standard tests, the progressive shift towards performance-based design has opened the door to use of advanced methods based on numerical models. These approaches will not replace standard testing, but they can already be used in a complementary fashion, to extend the application of test data.

Some simplified modelling methods have also been established, such as the protected member equation in Eurocode 3 (EC3) [1], but as with all semi-empirical methods the results will tend to be conservative and there are of necessity a number of simplifying assumptions. CFD-based methodologies can in principle provide a much more detailed description of the thermal environment and the effects of localised heating, which could be used in conjunction with thermal analysis models to examine structural performance.

There are currently various approaches used to link the CFD-based models and the thermal analysis models. For example a range of advanced methodologies have been developed to couple the CFD model with the thermal and structural model, such as those examined in the FIRESTRUC [2] project with code pairs ANSYS-CFX, FDS-ANSYS, VESTA-STELA etc. All these implementations have certain aspects of pros and cons. 
Prior to this work [3], a dedicated fine-mesh thermal modelling tool, known as STELA (Solid ThErmaL Analysis), has been implemented within the RANS CFD code SOFIE [4]. However, this research suggested that detailed thermal analysis of structural members in the context of simulations of full-scale building fires remains problematic. This is partly due to the difference of scale between the mesh which can be afforded for the fire and that required for the thermal analysis of the structure, a particular problem with structured meshes, and also the generally high computational demands for coupled analyses. Moreover, existing approaches, no matter how the thermal model is implemented into the CFD model, either in embedded manner within the CFD code such as STELA, or by more generalised couplings such as with FDS-ANSYS, VESTA-DIANA, are limited to a specific structural arrangement of interest since it is necessary to define all model parameters in advance. Simulations must be repeated if details such as the structural geometry or the thermal properties are changed, a very inefficient procedure.

A more general and flexible methodology has now been proposed, still within the context of a CFD fire simulation, as reported elsewhere $[5,6]$. This is based on computation of a set of "steel temperature field" parameters within the whole of the calculation domain, accommodating, by means of simultaneous calculations, both uncertainties in the input parameters and possible variants to the specification. Hence the need for repeat simulations is bypassed. Furthermore, by predicting the "virtual member" temperatures at each point in space the limitations of existing methods with regards to the position of the structural component are overcome. Considering the potentially great computational costs associated with the large numbers of thermal analysis calculations required (equal to the number of gas-phase cells times the number of variants studied in the simultaneous calculations), approximate methods are employed to reduce the full 3D thermal response problem down to treatments which are essentially $1 \mathrm{D}$ but which include appropriate representations of the heat transfer processes in the other dimensions to reconstruct a quasi-3D solution. The further development, in particular, the development of a modelling representation for the effects of intumescent performance in fire is also described here. This new generalised methodology has been implemented as a submodel within the SOFIE RANS CFD code and is known as GeniSTELA (Generalised Solid ThErmaL Analysis).

\section{METHODOLOGY DEVELOPMENT}

\subsection{Brief Description}

When protected steelwork is exposed to fire, heat is transferred to the structure through a layer of insulation. The transient heating response of the member can in principle be described using conventional methods based on numerical heat transfer. However, full 3D analyses impose great computational demands, due to the large numbers of cells required in order to adequately resolve the steep thermal gradients during the initial heating. Even if the computational resource is available, in simple deterministic models there is no direct mechanism to accommodate uncertainties in the thermal properties and member specification. To overcome these problems, with an appropriate balance between accuracy and tractability, a novel quasi-3D analysis methodology has been developed [5]. This is achieved by constructing a generalised 1D model and further considering the $2 \mathrm{D}$ or $3 \mathrm{D}$ effects within the heat transfer processes by appropriate approximations and corrections. The computations are performed in each gas-phase CFD cell in the computational domain. 


\subsection{Generalised 1D Model}

The generalised 1D model is constructed through analysing the heat transfer to and within an element in an idealised protected steel member assumed to be exposed to heat on two faces, as shown in Figure 1 below:

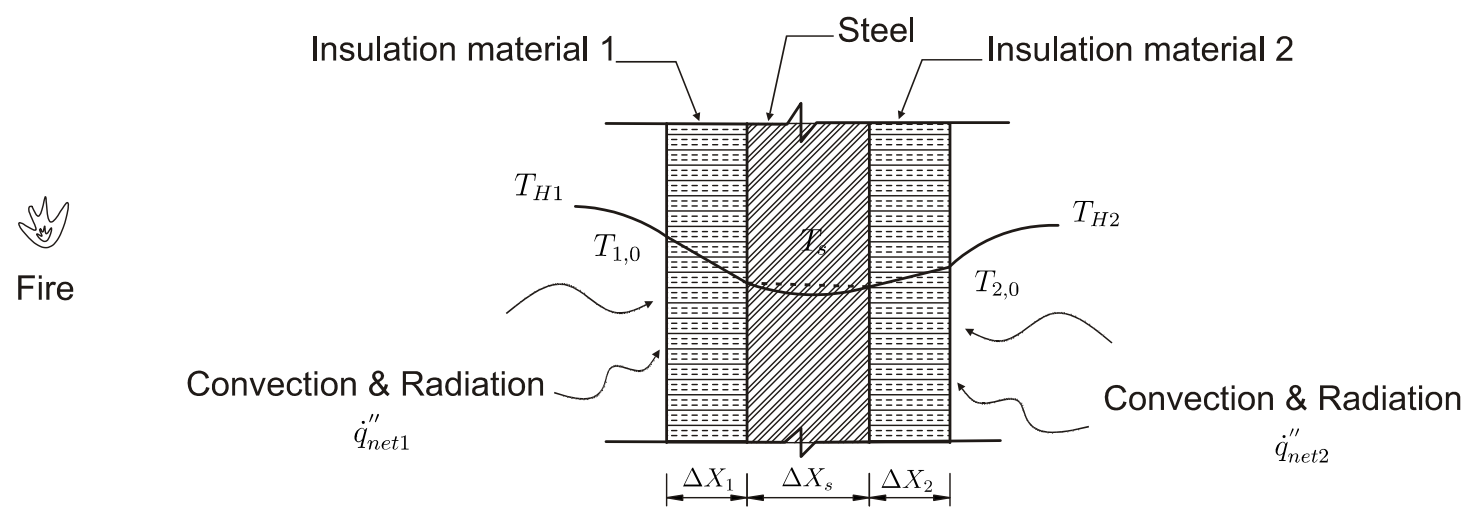

Figure 1. Schematic of Heat Transfer to Protected Steel Member

This element is supposed to be representative of a slice of a protected steel structure, e.g. a finite section of a flange or a web; two faces are used to allow for situations where the exposure conditions on each side might vary, encompassing also the case of hollow sections with very different exposures on the inside of the structure, though in that case the insulation thickness on the inside is reduced to zero.

The generalised 1D model provides a modelling framework which exploits a simple thermal penetration model for the protection coupled to an essentially lumped parameter representation of the steel heating. The governing equations for this model are derived by considering the net energy balance together with surface heat transfer boundary conditions [7] as given below:

Energy balance equation:

$$
\frac{\partial E_{\text {system }}}{\partial t}=\dot{q}_{n e t}^{\prime \prime}
$$

i.e.

$$
\begin{aligned}
& \rho_{S} \cdot c_{p s} \cdot \frac{\partial T_{S}}{\partial t} \cdot \Delta x_{S}+w_{p 1} \cdot \rho_{1} \cdot c_{p 1} \cdot \frac{\partial T_{1}}{\partial t} \cdot \Delta x_{1}+w_{p 2} \cdot \rho_{2} \cdot c_{p 2} \cdot \frac{\partial T_{2}}{\partial t} \cdot \Delta x_{2} \\
& =h_{c 1} \times\left(T_{H 1}^{(n)}-T_{1,0}^{(n)}\right)+\dot{q}_{r 1}^{\prime \prime}-\varepsilon_{m 1} \cdot \sigma \cdot T_{1,0}^{(n)_{4}}+h_{c 2} \times\left(T_{H 2}^{(n)}-T_{2,0}^{(n)}\right)+\dot{q}_{r 2}^{\prime \prime}-\varepsilon_{m 2} \cdot \sigma \cdot T_{2,0}^{(n)_{4}}
\end{aligned}
$$

The terms shown in the expanded equation here represent, respectively, the transient heating of the steel and protection layer on each side, and the convection, radiation and re-radiation for each surface of the protected member. A semi-empirical treatment is adopted for transient heating, allowing for spatially- and temporally-varying temperature gradients within the solid. The boundary conditions are supplied from the heat transfer solution for the surfaces, using the following equations:

$$
\dot{q}_{n e t 1}^{\prime \prime}=\frac{k_{1}}{w_{p 1} \Delta x_{1}} \cdot\left(T_{1,0}^{(n)}-T_{S}\right)
$$


i.e. $\quad h_{c 1} \times\left(T_{H 1}^{(n)}-T_{1,0}^{(n)}\right)+\dot{q}_{r 1}^{\prime \prime}-\varepsilon_{m 1} \cdot \sigma \cdot T_{1,0}^{(n)} 4=\frac{k_{1}}{w_{p 1} \Delta x_{1}} \cdot\left(T_{1,0}^{(n)}-T_{S}\right)$

$$
\dot{q}_{n e t 2}^{\prime \prime}=\frac{k_{2}}{w_{p 2} \Delta x_{2}} \cdot\left(T_{2,0}^{(n)}-T_{S}\right)
$$

i.e. $\quad h_{c 2} \times\left(T{ }_{H 2}^{(n)}-T_{2,0}^{(n)}\right)+\dot{q}_{r 2}^{\prime \prime}-\varepsilon_{m 2} \cdot \sigma \cdot T_{2,0}^{(n)} 4=\frac{k_{2}}{w_{p 2} \Delta x_{2}} \cdot\left(T_{2,0}^{(n)}-T_{S}\right)$

where:

$\sigma \quad$ is Stefan-Boltzmann constant $\left(5.67 \times 10^{-8} \mathrm{~W} / \mathrm{m}^{2} / \mathrm{K}^{4}\right)$;

$\dot{q}_{r 1}^{\prime \prime}, \dot{q}_{r 2}^{\prime \prime} \quad$ are incident heat fluxes on each side;

$T_{1,0}^{(n)}, T_{2,0}^{(n)} \quad$ are surface temperatures at gas/solid interfaces;

$T_{s}, T_{1}, T_{2} \quad$ are steel and average protection layer temperatures, respectively;

$h_{c 1}, h_{c 2} \quad$ are convection coefficients;

$\varepsilon_{m 1}, \varepsilon_{m 2} \quad$ are emissivities of protection layers;

$\rho_{s}, \rho_{1,} \rho_{2} \quad$ are densities of steel and protection layers, respectively;

$\Delta x_{s}, \Delta x_{1}, \quad$ are thicknesses of steel and protection layers, respectively;

$\Delta x_{2}$

$w_{p 1}, w_{p 2} \quad$ are weight factors of protection layers, defined in terms of the thermal

penetration depth of the protection, given in the form of Equation 4:

$w_{p}=\min \left\{\frac{A_{\text {actual }}}{A_{\text {model }}}=\frac{\delta}{\Delta x_{p}}, 1\right\}$

where

$$
\delta=2 \cdot\left(\frac{k_{p} \cdot t}{c_{p} \cdot \rho}\right)^{1 / 2}
$$

$c_{s}, c_{p 1}, c_{p 2} \quad$ are specific heat of steel and protection layers, respectively;

$k_{1}, k_{2} \quad$ are thermal conductivity parameters of protection layers.

The temperature/time dependent characteristics, including moisture and intumescence effects, are incorporated in certain parameters for generalisation of the methodology. The moisture effects were treated by varying the relevant thermal properties of the material, in particular, the specific heat and thermal conductivity, by means of an additional temperature-dependent source term in the energy equation representing latent heat effects. The treatment for moisture effects was described in detail in the previous paper [5] whilst intumescence is described in section 3 below.

It is well-known that the above situation is a strongly coupled problem, with the net heat fluxes at the gas-solid interface very much dependent on the surface temperature, but both also related to the transient thermal response of the structure itself. Numerical instabilities might become evident if inadequate solution procedures are used; these are overcome using a Newton-Raphson method to update the surface temperature from the heat transfer boundary condition governing equations and thereafter, with the updated surface temperature as a boundary condition, solving the overall energy balance equation (Eq. 1) with the Runge-Kutta method to obtain the steel temperature. 


\subsection{Quasi-3D Model}

Use of a fundamentally 1D treatment is essential, considering the costs of doing a full 3D analysis in every computational cell and including a sufficient number of parametric variations. However, adoption of a simple 1D model for thermal analysis could clearly lead to some modelling inaccuracies. These could in principle be in either direction, resulting in either conservative (over-design) or non-conservative (unsafe) results. The former aspect is not a major concern since the method is in any case far more flexible than other simple models, and by using generalised treatments conservatism is already greatly reduced. The latter aspect is a more obvious problem, and in order to overcome it, methods for treating important 2D and $3 \mathrm{D}$ effects are needed. A number of corrections factors have been implemented in the model, encompassing the factors indicated in Figure 2, i.e. the junction effect, end effect, heat sink effect and axial temperature gradient effect.

- Junction effect

This effect accounts for the effects of the connection between two different parts of a member under differential heating, i.e. where there is a temperature differential due to the fact that the exposure of one part of the structural component, e.g. a flange or a web, is dominant over that of a connected part.

- $\quad$ End effect

Along the length of the flange, the steel temperature changes, especially for the cells in the two ends of the flange, which might be a worst case position for temperature by virtue of the fact that they are exposed to heat arriving from two different directions, i.e. their heated surface is larger.

- Heat sink effect

It considers the thermal effects when a structural member is in contact with a ceiling slab.

- $\quad$ Axial temperature gradient

This effect accounts for the case when the structure passes from a cold layer into a hot layer, the temperature changes greatly with height, or where there are significant horizontal temperature gradients.

It is important to note that these effects are only critical where they negatively impact the performance of the member, i.e. increase the solid temperatures, and in the majority of cases the opposite is true, i.e. the default procedure is a good representation of the "worst" case. Thus, while it is vital to show that these possible corrections have been appropriately considered, their effect on the final results has been found to be fairly limited.

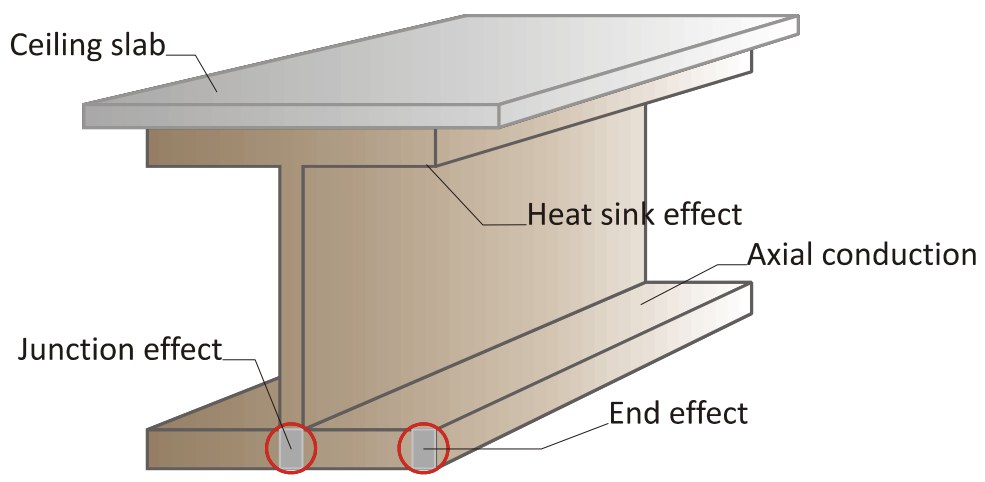

Figure 2. Cross-section of the Beam with Locations of Possible Correction Effects 


\section{MATERIAL PROPERTIES}

The aforementioned model might be considered as a reasonable representation of the fundamental aspects of the heat transfer phenomena. However, in practice, several factors are found to have a great impact on the transient response, in particular the thermal properties of the protection materials, which affect the surface temperature and thus the steel temperature. It is known that these properties are often strongly temperature/time dependant and the use of constant values may result in significant errors in some cases. The methodology developed here aims at generalising the thermal analysis to accommodate all important phenomena; conventional approaches to treatment of moisture effects have already been implemented, referencing modified specific heats and thermal conductivities [5]. This is now extended further to include the effects of intumescence, clearly of great practical relevance to the case of protected steelwork. In order to do so, geometrical and density variations must also be explicitly treated.

Intumescent materials are an increasingly popular form of fire protection, due to a number of advantages arising from the fact that they can be applied as thin, aesthetically pleasing, coatings either before or after construction $[8,9,10]$. When in contact with high temperatures, they will swell and form a layer of carbonaceous char which has much greater thickness than the initial state. The char subsequently acts as a thermal barrier to effectively protect the substrate against increase in temperature. Nevertheless, during the process of intumescence, the material properties are severely changed along with mass transport and endo- and exothermic reactions. These properties include thermal conductivity, specific heat, density and thickness of the intumescent layer.

Several research studies have been carried out to determine the effective intumescent thermal properties by experimental tests, in conjunction with some form of numerical analysis. These include bench-scale cone calorimeter tests and small-scale furnace tests on coated plates [11, 12], and furnace tests on cellular beams [13]. The first authors conducted studies on typical water-based and solvent-containing intumescent systems [10] and later on a high-performance material, i.e., epoxy resin containing boric acid and phosphate-based flame retardant [12]. The results from the former showed a significant slow down of temperature increase between $200-300^{\circ} \mathrm{C}$, due to intumescence, i.e. the formation of an insulating char and other co-acting energy absorbing processes; temperature influences during the latter tests also resolved a damping effect at $150^{\circ} \mathrm{C}$ due to the endothermic reaction of boric acid, which also releases water. Layer thickness effects were non-linear. Considering first the geometrical expansion, a simple conceptual model would suggest that thermal equivalence to a finite thickness problem can be achieved by simply scaling the thermal conductivity by the layer thickness, $d(=1 \mathrm{~m})$, giving an effective thermal conductivity, $k / d$. Density is scaled in the same way, and specific heat by the inverse of $d$, but these parameters always appear as factors of each other so these scalings vanish in the term $\rho c_{p}$.

The description of the temperature-dependent intumescent thickness, $d$, can be determined from an expression for the expansion ratio. We postulate that this will fit the general form:

\begin{tabular}{ll}
\hline$R=1$ & $T<T_{\text {lower }}$ \\
\hline$R=1+\frac{1}{2}\left(R_{f}-1\right)\left(\frac{T-T_{\text {lower }}}{T_{\text {mid }}-T_{\text {lower }}}\right)^{n}$ & $T_{\text {lower }} \leq T<T_{\text {mid }}$ \\
\hline$R=R_{f}-\frac{1}{2}\left(R_{f}-1\right)\left(\frac{T_{\text {upper }}-T}{T_{\text {upper }}-T_{\text {mid }}}\right)^{n}$ & $T_{\text {mid }} \leq T<T_{\text {upper }}$ \\
\hline$R=R_{f}$ & $T \geq T_{\text {upper }}$ \\
\hline
\end{tabular}


Where

$\begin{array}{ll}R & \text { is the time-dependent expansion factor; } \\ R_{f} & \text { is the final expansion factor; } \\ T_{\text {lower }}, T_{\text {upper }}, T_{\text {mid }} & \text { are the critical temperatures where scaling factor changes; } \\ T & \text { is the current averaged intumescent temperature; } \\ n & \text { is a shape factor power. }\end{array}$

Here, besides the relevant temperatures limits, the critical controlling parameters are the shape factor $n$ and the overall expansion ratio $R_{f}$. An approximate calibration has been performed by comparison with test data, including the results of Bartholmai [12] giving a value of $n=2$. Taking an approximate temperature range from the DTG results of the latter study, and assuming $R_{f}=10$, gives the following curve:

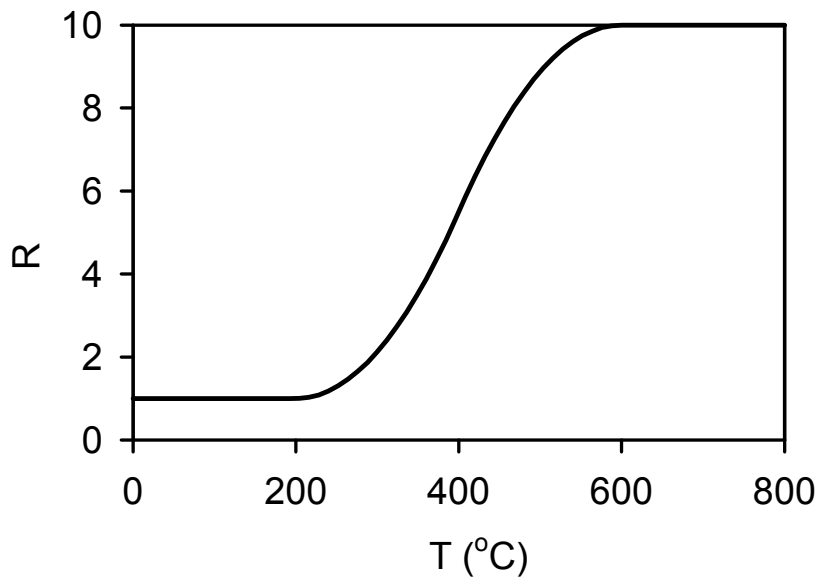

Figure 3. Scaling Factor R Change with Temperature

In fact, a variety of overall 1D expansion ratios have been reported in the literature, 10 [14] , 15-30[8], 50-220 [11].

The key parameter for the thermal model is the conductivity, or its scaled value, i.e. $k / d$. The conductivity itself is affected by fundamental changes in the material as it intumesces $[11,12,15]$. Unfortunately, the effect is non-linear and very dependent on initial thickness, and most pronounced at the smaller thicknesses typical of real applications; hence, there would appear to be no substitute for its direct experimental determination. A series of cone calorimeter tests hence have been carried out at the University of Edinburgh to investigate derivation of the temperature/time-dependent thermal properties of intumescent materials, as also reported previously [6]. Their intumescent expansion ratio was approximated by measuring peak char thickness after the test and their effective thermal conductivity deduced from calibration of a one-dimensional thermal model implemented in a spreadsheet. In the meanwhile, the various literature results would suggest an initial increase followed by a fall during intumescence and finally a sharp rise during material degradation. The final submodel for intumescent properties is constructed by combined consideration of the experimental findings with the literature results.

\section{MODEL VERIFICATION AND VALIDATION}

The above conceptual model has been implemented as a submodel called GeniSTELA within the SOFIE RANS CFD code [4]. Representative empirical values are adopted for some terms such as the initial conditions, the dry thermal properties, moisture content, etc., and their influence has been studied by exercising the model with different sets of input parameter values. The performance of 
the model was assessed by performing sensitivity studies, looking at the effects of a range of numerical and physical parameters. Comparisons were also made with the results from the EC3 protected member equation [1].

The case used for verification studies is the protected steel indicative, UC254x254/73, in the full-scale tests on a $12 \times 12 \mathrm{~m}$ compartment undertaken at BRE Cardington [16]; this member was protected with about $25 \mathrm{~mm}$ of Fendolite MII sprayed fibre (baseline $\rho=680 \mathrm{~kg} / \mathrm{m}^{3}, k=0.19 \mathrm{~W} / \mathrm{m} / \mathrm{K}$ ). Figure 5 shows the test compartment while Figure 6 shows the SOFIE temperature predictions.

In the test a variety of thermal parameter measurements were made, encompassing conditions in the gas phase (temperatures, velocities and heat fluxes) and in the solid phase (steel temperatures in protected beams, columns and indicatives, with and without protection); this study also serves for the validation of the model, comparing the model predictions with the measured steel temperatures in the protected indicative.

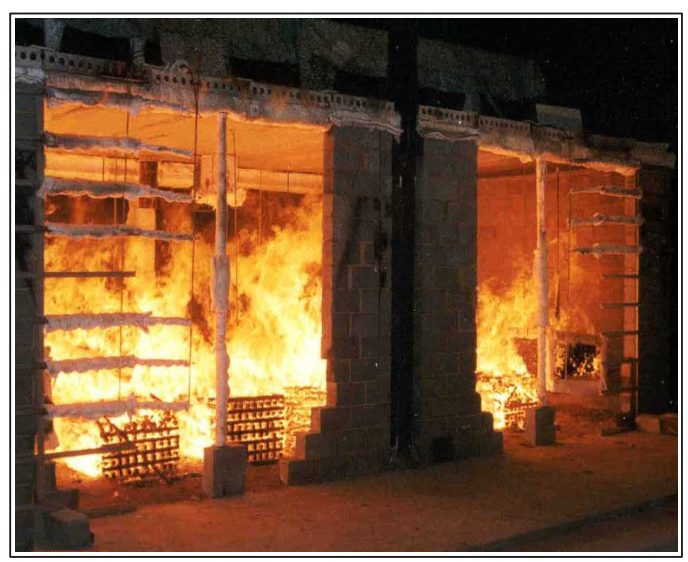

Figure 5. BRE Large Compartment Fire Test Figure 6. BRE Large Compartment Fire Modelling

\section{RESULTS}

\subsection{Simulation Results}

Gas and steel temperatures were computed using SOFIE and the coupled GeniSTELA code. In qualitative terms the results showed the expected differences in steel and gas temperature fields, with relatively higher steel temperatures within the depth of the compartment compared to the openings. This is consistent with the fact that the thermal exposures are more severe deeper into the fire [16], and the model predictions from GeniSTELA are heavily influenced by the radiative terms, $\dot{q}_{r}^{\prime \prime}$, derived directly from the CFD calculation.

Figure 7 shows the temperature predictions for the protected indicative within the compartment. There is a large temperature gradient across the protection. 


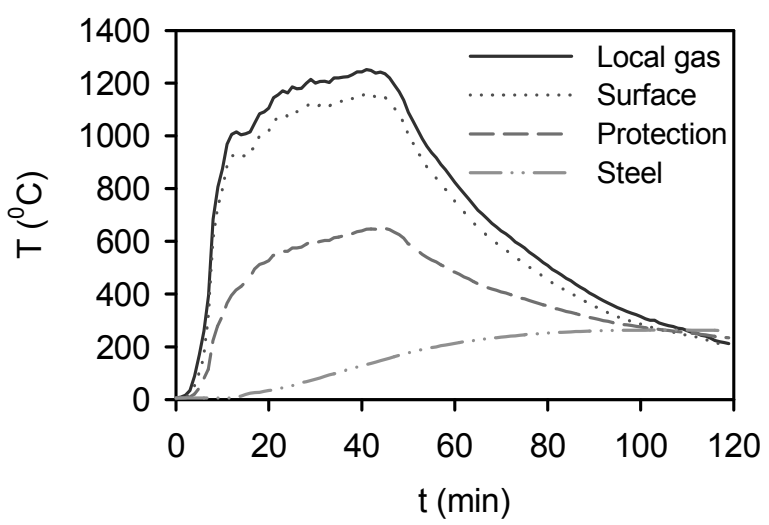

Figure 7. Temperatures at Protected Indicative, Test 8

Figure 8 shows a comparison of the predictions of steel temperature with the test together with EC3 prediction. The latter exceeds the measure temperature reflecting some conservatism in this semi-empirical method, while the prediction from GeniSTELA indicates a sufficient match with the test.

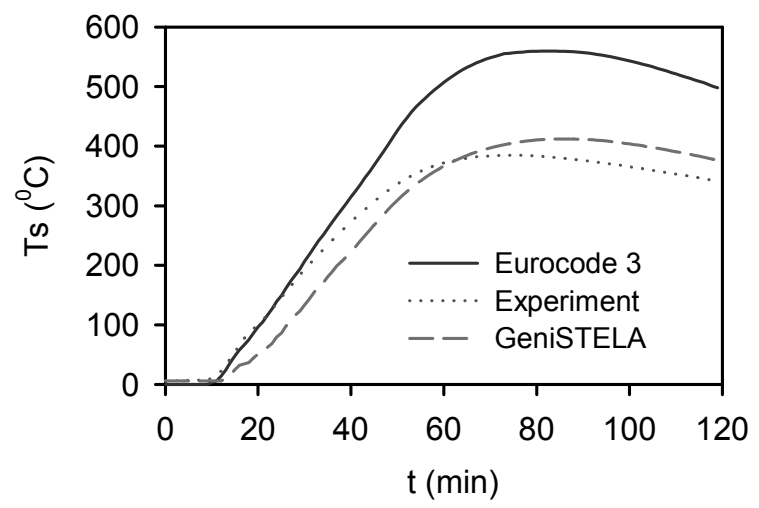

Figure 8. Comparison of Steel Temperatures

\subsection{Sensitivity Study Results}

Some results from the sensitivity study are shown in Figures $9 \sim 11$ for the effects of changing the steel flange thickness (spanning UC 254x254/73,107,167), the protection thickness (12.5 to 50mm) and the protection thermal conductivity $(0.1$ to 0.4$)$. The results for changing the protection thermal conductivity mirror the latter, and show the expected strong influence of protection properties.

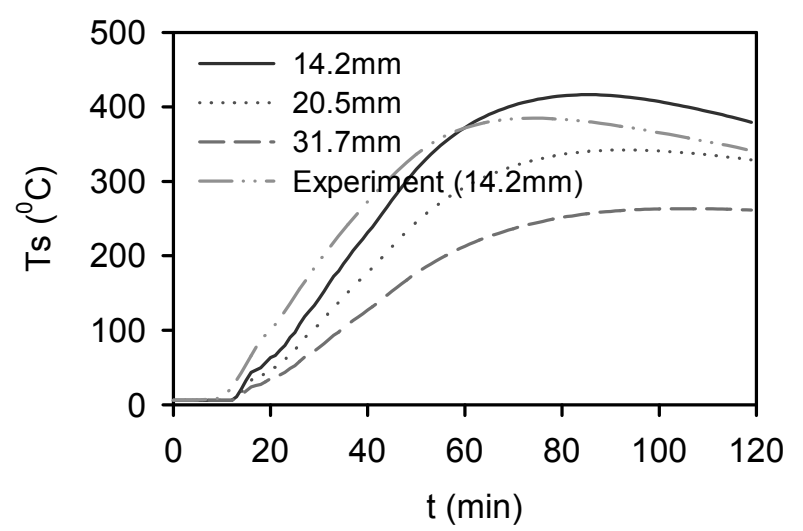

Figure 9. Effect of Flange Thickness on Steel Temperature 


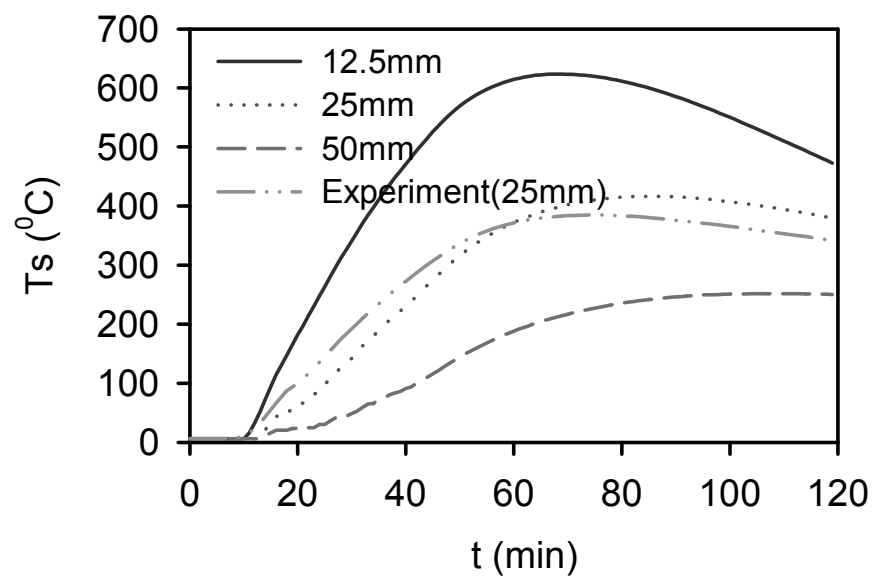

Figure 10. Effect of Protection Thicknesses on Steel Temperature

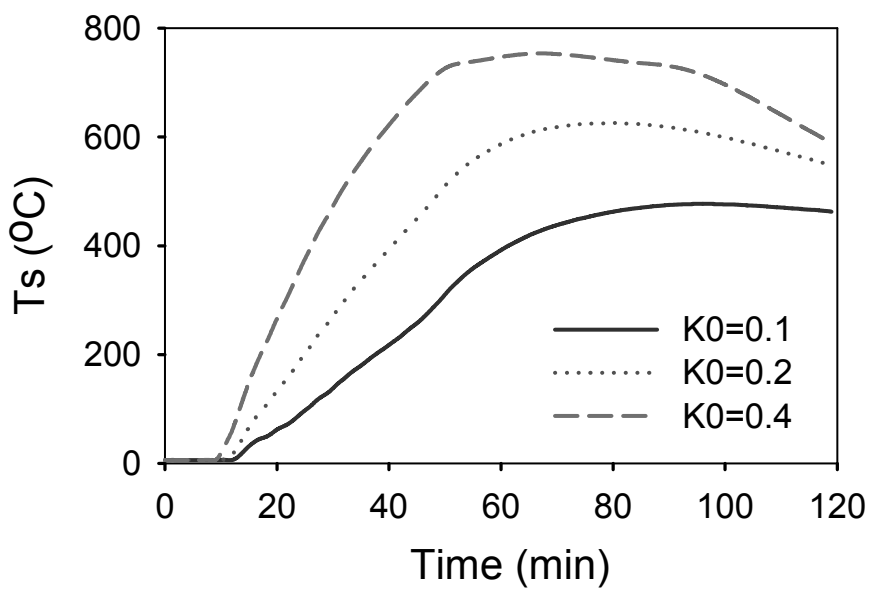

Figure 11. Effect of Protection Thermal Conductivity on Steel Temperature

Some other effects such as the moisture effect, the emissivity effect and the intumescent protection effect have also been examined during the case study. It is found that the moisture content could be an important factor within certain temperature range before the moisture has vanished. The emissivity effect has been studied by comparing steel temperature predictions from a range of emissivity values representing high emissivity (above 0.5 ) and low emissivity (below or equal to 0.5 ) either for the fire or for the member. It is found that the fire emissivity doesn't affect the final steel temperature prediction but that the member surface emissivity is a key factor that may influence the temperature rise in the steel. But overall, the emissivity has a low-order effect on performance especially for the start of the heating regime when convective heat transfer is dominant. This point is consistent with the findings by Staggs and Phylaktou [17]. The intumescent protection has strong effects on the final results regarding to the type of intumescent protection and protection thickness. Those interesting findings during the sensitivity study facilitate the further implementation of GeniSTELA results into a newly developed code called GeniSTRUC (Generalised STRUCtural Analysis) [18] for a generalised analysis of structural mechanical responses. Here, the GeniSTELA calculated steel temperature at each cell location of the structural member would be implemented via GeniSTRUC to the related location for structural analysis. The initial GeniSTRUC results indicate that normally the failure happens as expected in the middle cell of the beam but not in the end cell. This point demonstrates the practical usage of GeniSTELA in the structural fire design field. 


\section{COMPUTATIONAL REQUIREMENTS STUDY}

In order to examine the practical use of this methodology, the overall computational requirements have been assessed in terms of the CPU time usage. Also, the potential for reducing the frequency of the calls to the GeniSTELA steel temperature solver has been explored by changing this from the default of once every 10 iterations. This default was determined to match the usual frequency of calls to the radiation solver, since one of the key drivers of the thermal response is the radiation field and in most cases there is no benefit in recomputing steel temperatures if this has not been updated. The change in GeniSTELA call frequency is realised by introducing a timestep factor variable (tfactor) in the model in order to increase the intervals between calls.

The results showed that GeniSTELA uses around $1 \%$ of the CPU time for the flow solver, including radiation, when called at the default interval of 10 iterations. Simulations were then undertaken with a tfactor value of 10 for a localised fire scenario, with a constant fire size, having realistic steel temperature increases in 10 minutes. Figure 12 shows the results for the respective steel temperature predictions, confirming that even when called only once per every 100 main solver iterations, i.e. with just six calls of the GeniSTELA solver altogether, there is a very small effect on the final steel temperature result, especially at latter times. Figure 13 shows the evolution of the percentage temperature difference. It is obvious that the discrepancy is only important in the early stage, with the maximum difference being only of order $8 \%$, mostly within $3 \%$. Using an intermediate value of tfactor $=2$ gave results much closer to the default case, with a maximum discrepancy of only $1.5 \%$.

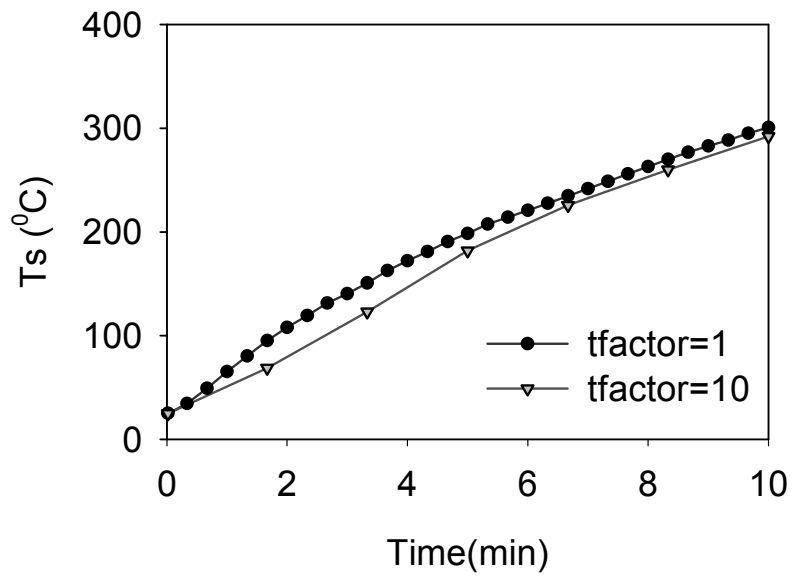

Figure 12. Predicted Steel Temperatures with $t$ factor $=1$ and 10

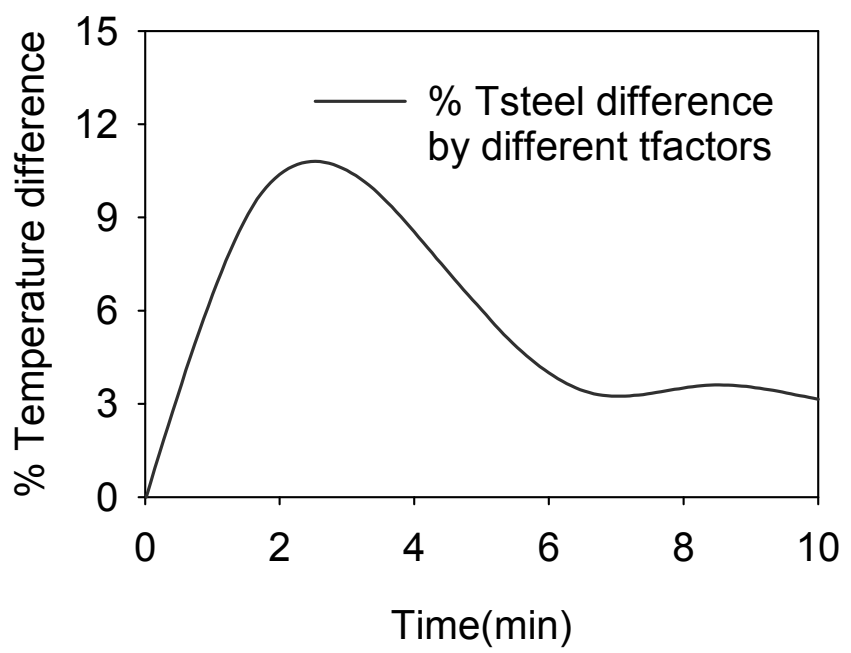

Figure 13. Difference in Steel Temperature Against Time Using tfactor $=1$ and 10 
The above findings are of course only of relevance for this particular steady fire, and for more general cases, where the heat release rate may be changing rapidly, higher frequencies may be required. In practice, the frequency of the GeniSTELA call could be adjusted by automatic selection linked to heating rates, in order to achieve the best efficiency. Nevertheless, this initial study suggests that a full set of parametric calculations (10-100 cases) could be afforded without any significant compromise of accuracy, before the GeniSTELA analysis becomes the dominant part of the computation.

\section{CONCLUSIONS}

A generalised methodology for thermal analysis of protected steel structures in fire is described. A framework for the inclusion of treatments for intumescence effects has been established, with provision for simple calibration in the case of each specific formulation of interest. The GeniSTELA implementation of the method is based on simultaneous computations spanning the range of cases of interest, providing a novel generalised methodology. The results confirm the sufficiency of the algorithms adopted and comparisons with measurements in a post-flashover compartment fire test are satisfactory. Computational demands are found to be acceptable. Strong dependencies on the thermal properties of the protection materials are observed in the sensitivity studies. These results serve to illustrate the importance of using generalised methodologies in tackling thermal response problems and suggest a new approach to structural fire safety engineering design.

\section{ACKNOWLEDGEMENTS}

The first author acknowledges the support of her industrial sponsor, the BRE Trust, and the School of Engineering. Thanks also to the members of BRE Centre for Fire Safety Engineering, and technical assistants, for their support during the research. Useful comments on intumescent properties were received from Yong Wang and Matthias Bartholmai, and Daniel Joyeux and Mario Fontana via IAFSS email list.

The work reported in this paper has formed part of FireGrid, www.firegrid.org. This project was/is co-funded by the Technology Strategy Board's Collaborative Research and Development programme, following an open competition.

\section{NOMENCLATURE}

$c_{p s}, c_{p 1}, c_{p 2} \quad$ specific heat capacity of steel and protection layers 1,2 , respectively $(\mathrm{J} / \mathrm{kg} / \mathrm{K})$

$d \quad$ intumescent layer thickness (m)

$E_{\text {system }} \quad$ total energy in the system $(\mathrm{J})$

$h_{c 1}, h_{c 2} \quad$ convection coefficient on sides 1,2 , respectively $\left(\mathrm{W} / \mathrm{m}^{2} / \mathrm{K}\right)$

$k_{1}, k_{2} \quad$ thermal conductivity of protection layers 1,2 , respectively $(\mathrm{W} / \mathrm{m} / \mathrm{K})$

$\dot{q}_{n e t 1}^{\prime \prime}, \dot{q}_{n e t 2}^{\prime \prime} \quad$ net heat flux on sides 1,2 , respectively $\left(\mathrm{W} / \mathrm{m}^{2}\right)$

$\dot{q}_{r 1}^{\prime \prime}, \dot{q}_{r 2}^{\prime \prime} \quad$ incident heat flux on sides 1,2 , respectively $\left(\mathrm{W} / \mathrm{m}^{2}\right)$

$t \quad$ time (s)

$T_{H 1}, T_{H 2} \quad$ gas temperature on sides 1,2 , respectively $(\mathrm{K})$

$T_{1,0}^{(n)}, T_{2,0}^{(n)} \quad$ surface temperature at gas/solid interface on sides 1,2 at $n^{\text {th }}$ time-step, respectively $(\mathrm{K})$ 


$\begin{array}{ll}T_{s}, T_{1}, T_{2} & \text { average temperatures of steel and protection layers on sides } 1,2 \text {, respective } \\ & \text { (K) } \\ w_{p 1}, w_{p 2} & \text { "weight factors" of protection layers on sides } 1,2 \text {, respectively }(-) \\ \Delta x_{s}, \Delta x_{1}, \Delta x_{2} & \text { thickness of steel and protection layers on sides } 1,2 \text {, respectively }(\mathrm{m}) \\ \delta_{1}, \delta_{2} & \text { thermal penetration depth on sides } 1,2 \text {, respectively (m) } \\ \varepsilon_{m 1}, \varepsilon_{m 2} & \text { emissivity of protection layers on sides } 1,2 \text {, respectively (-) } \\ \rho_{s}, \rho_{1}, \rho_{2} & \text { density of steel and protection layers on sides } 1,2 \text {, respectively }\left(\mathrm{kg} / \mathrm{m}^{3}\right) \\ \sigma & \text { Stefan-Boltzmann constant }\left(5.67 \times 10^{-8} \mathrm{~W} / \mathrm{m}^{2} / \mathrm{K}^{4}\right)\end{array}$

\section{REFERENCES}

[1] British Standards Institution, "Eurocode 3: Design of Steel Structures - Part 1-2: General Rules - Structure Fire Design", 2002.

[2] Welch, S., Miles, S. Kumar, S., Lemaire, T. and Chan, A., "FIRESTRUC - Integrating Advanced Three-dimensional Modelling Methodologies for Predicting Thermo-mechanical Behaviour of Steel and Composite Structures Subjected to Natural Fires", Proceedings of $9^{\text {th }}$ International IAFSS Symposium, 2008, Karlsruhe, Germany.

[3] Kumar, S., Welch, S., Miles, S.D., Cajot, L.-G., Haller, M., Ojanguren, M., Barco, J., Hostikka, S., Max, U. and Röhrle, A., "Natural Fire Safety Concept - The Development and Validation of a CFD-based Engineering Methodology for Evaluating Thermal Action on Steel and Composite Structures", European Commission Report EUR 21444 EN, 2005, Vol. 150 pp. ISBN 92-894-9594-4.

[4] Lewis, M.J., Moss, J.B. and Rubini, P.A., "CFD Modelling of Combustion and Heat Transfer in Compartment Fires", Proceedings of $5^{\text {th }}$ International Symposium on Fire Safety Science, 1997, pp. 463-474.

[5] Liang, H. and Welch, S., "A Novel Engineering Tool for Thermal Analysis of Structural Members in Natural Fires", Proceedings of $4^{\text {th }}$ International Workshop on Structures in Fire, 2006, Aveiro, Portugal.

Also available: $h$ ttp://hdl.handle.net/1842/1985

[6] Liang, H., Welch, S. Stratford, T. and Kinsella, E.V., "Development and Validation of a Generalised Engineering Methodology for Thermal Analysis of Structural Members in Fire", Proceedings of $5^{\text {th }}$ International Seminar Fire \& Explosion Hazards, 2007, Edinburgh, UK.

Also available: http://hdl.handle.net/1842/1990

[7] Carslaw, H.S. and Jaeger, J.C., "Conduction of Heat in Solids", Oxford University, 1959.

[8] Goode, M.G. (ed.), "Fire Protection of Structural Steel in High-rise Buildings", NIST GCR 04-872, 2004.

[9] Jimenez, M., Duquesne, S. and Bourbigot, S., "Characterisation of the Performance of An Intumescent Fire Protective Coating”, Surface and Coatings Technology, 2006, Vol. 201, issue 3-4, pp. 979-987.

[10] Bailey, C.G., "Advances in Fire Engineering Design of Steel Structures", Proceedings of ICE, Structures and Buildings, 2006, Vol. 159, issue SBI, pp. 21-35.

[11] Bartholmai, M., Schriever, R. and Schartel, B., "Influence of External Heat Flux and Coating Thickness on the Thermal Properties of Two Different Intumescent Coatings using Cone Calorimeter and Numerical Analysis", Fire and Materials, 2003, Vol. ?????, pp. 151-162.

[12] Bartholmai, M. and Schartel, B., "Assessing the Performance of Intumescent Coatings using Bench-scaled Cone Calorimeter and Finite Difference Simulations", Fire and Materials, 2007, Vol. 31, pp. 187-205. 
[13] Bailey, C.G., "Indicative Fire Tests to Investigate the Behaviour of Cellular Beams Protected with Intumescent Coatings", Fire Safety Journal, 2006, Vol. 36, No. 8, pp. 689-700.

[14] Desanghere, S. and Joyeux, D., "Development of Design Rules for the Fire Behaviour of External Steel Structures", ECSC Project No. 7210-PR-380, Final Report, 2005.

[15] Tan, K.H., Wang, Z.H. and Au, S.K., "Heat Transfer Analysis for Steelwork Insulated by Intumescent Paint Exposed to Standard Fire Conditions", Proceedings of $3^{\text {rd }}$ International Workshop on Structures in Fire, 2004, Ottawa, Canada.

[16] Welch, S., Jowsey, A., Deeny, S., Morgan, R. and Torero, J.L., "BRE Large Compartment Fire Tests - Characterising Post-flashover Fires for Model Validation", Fire Safety Journal, 2007, Vol. 42, pp. 548-567.

[17] Staggs, J. and Phylaktou, H., "The Effects of Emissivity on the Performance of Steel in Furnace Tests", Fire Safety Journal, 2008, Vol. 43, pp. 1-10.

[18] Liang, H., Welch, S., Faure, L., and Gillie, M., "Proceedings of International Conference on Applications of Structural Fire Engineering”, 2009, Prague, Czech Republic. 